\title{
Twitter como instrumento para fomentar la participación del profesorado en los cursos formativos
}

Twitter as an instrument to encourage the participation of teachers in training courses

\section{Elena Berrón Ruiz}

Centro de Formación del Profesorado e Innovación Educativa de Ávila

eberron@educa.jcyl.es

María Victoria Régil López

Centro de Formación del Profesorado e Innovación Educativa de Ávila

mvregil@educa.jcyl.es

Fecha presentación: 19/09/2018 | Aceptación: 22/03/2018 |Publicación: 22/06/2018

\begin{abstract}
Resumen
La creciente incorporación de las nuevas tecnologías en el sistema educativo demanda una revisión profunda en los procesos de gestión de los centros formativos, mejorando su presencia en las redes sociales. La investigación recogida en este artículo, de carácter cualitativo, presenta un estudio de caso realizado en el Centro de Formación del Profesorado e Innovación Educativa de Ávila (España) y persigue dos objetivos: el primero consiste en valorar la utilidad de distintas estrategias para dinamizar y difundir los cursos formativos a través de Twitter, mientras que el segundo pretende analizar el impacto que ha tenido dicha difusión en el incremento de la participación del profesorado. Los resultados muestran que las novedades introducidas en la dinamización han despertado el interés de los docentes, aumentando las interacciones realizadas en la red social y produciéndose un notable ascenso de su participación en los cursos.
\end{abstract}

Palabras clave: formación de profesores; curso de formación; nuevas tecnologías

\section{Resum}

La creixent incorporació de les noves tecnologies en el sistema educatiu demana una revisió exhaustiva sobre els procesos de gestió de la formació dels centres, millorant la seua presència a les xarxes socials. La investigació recollida en aquest article, de caràcter qualitatiu, presenta un estudi de cas en el Centre de Formació del Professorat i Innovació Educativa d’Àvila (Espanya) i persegueix dos objectius: el primer consisteix en valorar la utilitat de distintes estratègies per dinamitzar i difondre els cursos formatius a través de Twitter, mentre que el segon pretén analitzar l'impacte que ha tingut l'esmentada difusió en l'increment de la participació del professorat. Els resultats mostren que les novetats introduïdes en la dinamització han despertat l'interès dels docents, augmentant les interaccions realitzades en la xarxa social i produint-se un notable ascens de la seua participació en els cursos.

Paraules clau: formació del professorat; curs de formació; noves tecnologies

\section{Abstract}

The increasing incorporation of new technologies in the education system demands a deep revision in the management processes of the training centers, improving their presence in social networks. The qualitative research presented in this article presents a case study carried out at the Training Center of Teachers and Educational Innovation of Avila (Spain) and pursues two objectives: the first consists in value the usefulness of different strategies to boost and disseminate the training courses through Twitter, while the second seeks to analyze the impact that such dissemination has been on the participation of teachers. The results show that the innovations introduced in the dynamization have aroused the interest of the teachers, increasing the interactions made in the social network and producing a remarkable rise of their participation in the courses.

Key Words: teacher education; training course; new technologies 
Berrón Ruiz, Elena y Régil López, María Victoria (2018). "Twitter como instrumento para fomentar la participación del profesorado en los cursos formativos". @tic revista d'innovació educativa, 20, 43-51.

\section{Introducción}

La formación permanente del profesorado es uno de los elementos clave en la calidad educativa y debe asegurar la conexión entre la educación y la sociedad, actuando como mecanismo que garantice el desempeño competente y apropiado del docente (Ramírez, 2015). El interés por la formación permanente del profesorado es muy antiguo pero, tal y como indican Manzanares y Galván-Bovaira (2012: 2), "la consciencia de que este conocimiento debe analizarse y actualizarse es mucho más reciente si lo comparamos con otros ámbitos de investigación educativa".

Para poder dar respuesta tanto a las finalidades generales del sistema educativo como a las necesidades específicas de los distintos colectivos docentes, anualmente los centros de formación del profesorado recogen las inquietudes que tienen los profesores para completar su formación (Bolívar, Domingo, Escudero, González y García, 2007). En Castilla y León (España), estos centros se denominan Centros de Formación del Profesorado e Innovación Educativa (en adelante, CFIE). Los CFIE son los encargados de impulsar, gestionar y posibilitar la formación permanente del profesorado de las enseñanzas no universitarias que están sostenidas con fondos públicos. Para ello, se ofrecen distintas modalidades formativas: cursos, seminarios, grupos de trabajo, proyectos de formación en centros, proyectos de innovación educativa y experiencias de calidad.

De todas ellas, en este artículo nos ocuparemos de los cursos, que constituyen una modalidad formativa que desarrolla contenidos científicos, didácticos, técnicos, culturales y pedagógicos, y en la que los profesores se inscriben individualmente según sus propios intereses educativos (Gairín y Armengol, 2008).

El CFIE de Ávila está situado en la capital, tiene un ámbito provincial y depende funcionalmente de su Dirección Provincial de Educación. La provincia de Ávila tiene una extensión de $8.051,15 \mathrm{~km}^{2}$, se encuentra al sur de Castilla y León y está formada por 248 municipios. Para llevar a cabo su Plan Provincial de Formación, el CFIE de Ávila se compone de siete asesorías de las siguientes áreas: convivencia, científico-tecnológica, lingüístico-comunicativa, educación física y artística, tecnologías de la información y la comunicación, social y matemáticas.

Por otra parte, durante la última década, nuestra sociedad se ha visto inmersa en una revolución tecnológica que ha supuesto un gran cambio en todos los ámbitos, consiguiendo tener un gran impacto en la educación (Davidson y Goldberg, 2009; Rodríguez, 2011). Las tecnologías de la información y la comunicación posibilitan un acercamiento rápido al conocimiento y sus lenguajes están modelando nuevos estilos cognitivos y otras formas de percepción de la realidad. Las redes sociales se utilizan para compartir hallazgos científicos e impedir que el conocimiento nuevo se quede estático (Levis, 2011) y cada vez es más numeroso el profesorado que reconoce su potencial en el aula (Grosseck, 2009; Trigueros, Sánchez y Vera, 2012).). De forma resumida, podemos decir que, en el mundo educativo, las redes sociales facilitan el feedback inmediato, la posibilidad de compartir información y documentación y la reflexión sobre determinados conceptos (Abella y Delgado 2015). En consecuencia, consideramos imprescindible que las instituciones educativas asuman la tarea de la alfabetización en estos nuevos lenguajes.
Twitter es actualmente una de las redes sociales y plataformas de comunicación más populares del mundo, presente en los cinco continentes y utilizada por más de 200 millones de usuarios. La importancia de esta poderosa red social es poner en contacto a un sinfín de personas y entidades a través de términos o hashtags que son fáciles de encontrar, ya que, utilizando las palabras de Pérez-Martínez, Rodríguez y Tobajas (2017: 682) "su estructura narrativa e interactiva facilita la comunicación entre sus usuarios". Creada en el 2006, Twitter se lanzó como una plataforma en la que se utilizaban inicialmente 140 caracteres (280 en la actualidad) para escribir mensajes, ideas, invitaciones, servicios o productos, que reciben el nombre de tweets. Dicha red social permite seguir y ser seguido por otras personas, facilitando la interacción gracias a la posibilidad de responder a otros o de mandar mensajes directos. El uso de Twitter dentro del mundo de la educación se ha popularizado y cada vez son más los docentes que lo utilizan en sus actividades con los alumnos o como parte de sus entornos personales de aprendizaje (Grosseck y Holotescu, 2011), ya que tiene una estructura colaborativa, permite la incorporación de contenidos multimedia y favorece la interactividad (Blanco y Sueiro, 2014).

La importancia de Twitter tiene que ver básicamente con la forma de comunicar ideas, proyectos o pensamientos de una manera particular y diferente a las demás redes sociales. Tal es su importancia que numerosos famosos, empresas y marcas han decidido crear cuentas oficiales desde las cuales promocionar sus productos, contar sus vidas privadas o asegurarse la llegada a miles de usuarios con los cuales tener contacto directo. También son muchos los docentes que aprovechan Twitter como una herramienta para potenciar su actividad profesional, permitiéndoles compartir, participar, colaborar, debatir, conversar, trabajar y crear con otros profesionales de la enseñanza, sin las limitaciones físicas que les impone su propio centro educativo. Asimismo, consideramos que los centros de formación del profesorado pueden y deben aprovechar las facilidades que proporciona esta red social para incrementar su presencia e impacto en la sociedad (Forkosh-Baruch y Hershkovitz, 2012).

Por todo lo expuesto, teniendo en cuenta las tendencias sociales actuales y apostando por la innovación, la calidad y las nuevas tecnologías, el equipo de asesores del CFIE de Ávila consideró conveniente desarrollar un conjunto de acciones secuenciadas que facilitaran la dinamización de los cursos formativos a través de Twitter, con el fin de motivar al profesorado y fomentar su participación. Las líneas de actuación son las decisiones que determinan el camino a seguir para alcanzar las metas propuestas (Imbernón 2007) y, en este artículo, realizado por dos de las asesoras, se describen las estrategias dinamizadoras que se han llevado a cabo a través de dicha red social y se analizan los resultados que han generado, lo cual nos ha permitido valorar su pertinencia para mejorar el desempeño de nuestras funciones.

\section{Metodología}

\subsection{Método y objetivos de la investigación}

El estudio realizado es de tipo analítico y comparativo, utilizando una metodología cualitativa (Barba, GonzálezCalvo y Barba-Martín, 2014; Stringer, 2007), ya que se buscaba analizar cómo había sido la difusión de los 
Berrón Ruiz, Elena y Régil López, María Victoria (2018). "Twitter como instrumento para fomentar la participación del profesorado en los cursos formativos". @tic revista d'innovació educativa, 20, 43-51.

cursos de formación permanente a través de Twitter durante los dos últimos años académicos (septiembre del 2015/junio del 2016 y septiembre del 2016/junio del 2017), y se ha comprobado su repercusión diferencial en la participación del profesorado, estableciéndose una comparación entre ambos. De forma más específica, los objetivos de la investigación se concretan en los dos siguientes:

1. Valorar la utilidad de distintas estrategias para dinamizar los cursos de formación permanente del profesorado en la red social de Twitter.

2. Analizar el impacto de la difusión realizada a través de Twitter en la participación del profesorado en los cursos formativos.

El método de investigación utilizado ha sido el estudio de caso, el cual se ha considerado como el más apropiado porque permitía realizar el "análisis de un problema determinado, dentro de un grupo y situación real, extraída de la propia experiencia de los sujetos que lo componen" (García Llamas, 2003: 93). Asimismo, siguiendo a Stake (1998), se ha llevado a cabo un estudio de caso de tipo intrínseco, que hace referencia a conocer un caso que despierta interés por alguna razón específica y que genera un informe descriptivo.

La población estudiada ha sido el profesorado no universitario que se encuentra en activo en los centros educativos sostenidos con fondos públicos en la provincia de Ávila, que es a quien va dirigida la formación permanente que ofrece nuestro CFIE. Dichos profesores suman un total de 2.250 y están distribuidos entre los siguientes tipos de centros (figura 1):

\begin{tabular}{lccc}
\hline & Públicos & Concertados & $\begin{array}{c}\text { Colegios Rurales } \\
\text { Agrupados (C.R.A.) }\end{array}$ \\
\hline Centros de Infantil y Primaria & 60 & 8 & 27 \\
Centros de Secundaria & 23 & 7 & \\
Centros de Formación Profesional & 1 & 1 & \\
Centros de Educación Especial & 1 & & \\
Conservatorio Profesional de Música & 1 & & \\
Escuela de Arte & 1 & & \\
Escuelas de Idiomas & 2 & & \\
Escuelas de Adultos & 6 & & \\
Equipos de Orientación Educativa & 6 & & \\
\hline
\end{tabular}

Figura 1. Centros educativos sostenidos con fondos públicos de la provincia de Ávila. Fuente: Elaboración propia

No obstante, cabe destacar que lo que interesa en la investigación desarrollada es "la riqueza y significatividad de la información, no la cantidad ni su estandarización" (Hernández, 2006: 21), puesto que "el objetivo primordial del estudio de un caso no es la comprensión de otros; la primera obligación es comprender este caso" (Stake, 1998: 17).

\subsection{Actuaciones de dinamización y difusión realizadas}

En este apartado se recogen las distintas estrategias utilizadas para difundir, promocionar y dinamizar los cursos formativos a través de la red social de Twitter. Cabe aclarar que, cuando hablamos de dinamizar nuestra cuenta, no nos referimos a intentar lograr, simplemente, el mayor número de seguidores posible sino que, además, buscamos que el profesorado al que van dirigidos nuestros cursos esté pendiente y deseoso de saber qué información nueva vamos a publicar y que interaccione con ella, comentándola y retwitteándola para enriquecerla y aumentar su difusión, ayudándonos, de este modo, a dar mayor valor y visibilidad a nuestros mensajes.

Las distintas estrategias utilizadas para difundir, promocionar y dinamizar los cursos formativos a través de la red social de Twitter son las que se enumeran a continuación:

- Manual sobre el uso de Twitter. Se elaboró un atractivo y sencillo manual para familiarizar al profesorado con Twitter y se difundió por todos los centros educativos de la provincia de Ávila. En dicho manual se recogía su definición y se ofrecían explicaciones sobre cómo se accede, terminología propia de dicha red social, las opciones que ofrece y algunos consejos para su correcta utilización. La figura 2 muestra un fragmento del mismo.

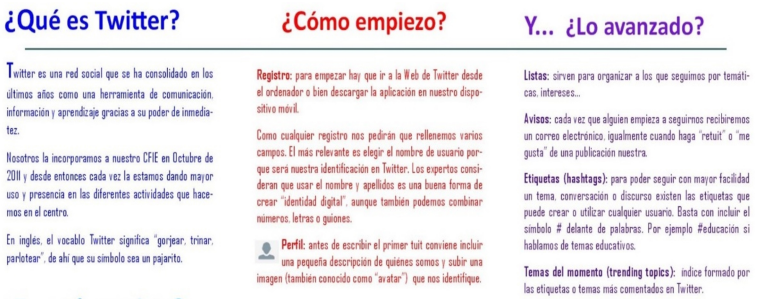

Figura 2. Fragmento del manual informativo sobre la utilización de Twitter Fuente: Elaboración propia

- Edición de videos promocionales para presentar los cursos en Twitter. Se utilizó el software online PowToon (disponible en www.powtoon.com), que permite crear vídeos didácticos y promocionales muy atractivos con facilidad y en poco tiempo. A modo de ejemplo, se recogen dos de los videos creados y difundidos posteriormente en Twitter:

- Curso Aplicaciones didácticas del cine (https://goo.gl/scVjvb)

- Curso Educación Física y hábitos saludables (https://goo.gl/oXhqnP)

- Fotografiado y grabación de actividades realizadas en los cursos y su difusión en la red social. Con la autorización previa de los ponentes y de los asistentes, durante los cursos se realizaron fotografías y pequeños videos y se fueron difundiendo en Twitter a tiempo real. Se puede acceder a ellos en el apartado "multimedia" de la cuenta del CFIE de Ávila (https://twitter.com/CFIE_Avila/media), tal y como muestra la figura 3 .

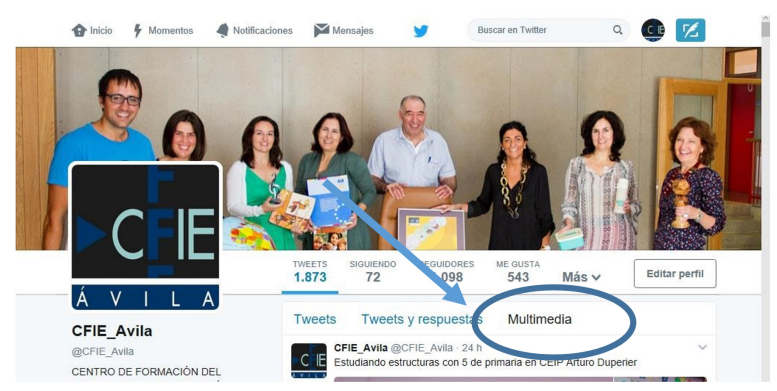

Figura 3. Indicación del acceso al apartado multimedia de la cuenta de Twitter. Fuente: https://twitter.com/CFIE_Avila/media 
Berrón Ruiz, Elena y Régil López, María Victoria (2018). "Twitter como instrumento para fomentar la participación del profesorado en los cursos formativos". @tic revista d'innovació educativa, 20, 43-51.

- Consulta de los momentos de máxima visibilidad para hacer publicaciones en Twitter. Se han utilizado distintas aplicaciones analíticas on-line que han permitido conocer los momentos más idóneos para realizar publicaciones. Entre dichas aplicaciones cabe destacar Analytics de Twitter y otras externas, también gratuitas, como Tweriod (disponible en: http://www.tweriod.com/) y Tweetstats (disponible en: http://www.tweetstats.com/). Tweriod remite información sobre la actividad de nuestra cuenta en la red social, proporcionando datos muy útiles como las franjas horarias más activas y con mayor número de seguidores conectados, o los contenidos que despiertan un mayor interés. Su presentación en gráficos es muy visual y permite comparar estos indicadores en distintos días de la semana, distinguiendo entre laborables y festivos. Así, por ejemplo, en la figura 4 observamos que, durante los días laborables, el mayor número de seguidores conectados se registra entre las 3 y las 5 de la tarde, coincidiendo con el momento en el que muchos usuarios están de reposo y aprovechan para consultar e interaccionar en Twitter. Respecto a Tweetstats, utiliza indicadores muy similares a los comentados en la aplicación anterior, pero su diferencia fundamental es que permite analizar datos de cualquier cuenta de Twitter, no sólo de la nuestra, por lo que no hace falta autentificarse.

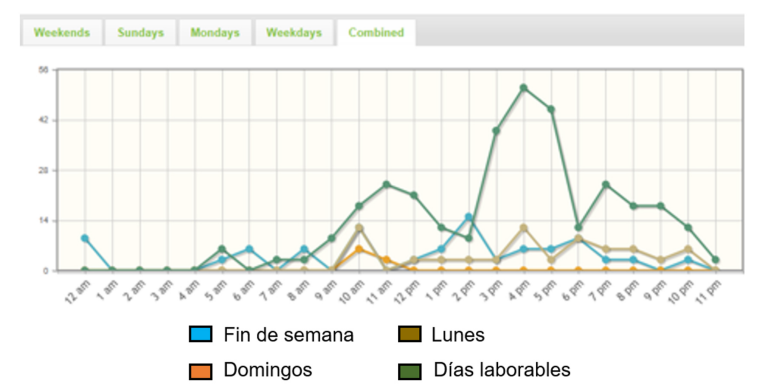

Figura 4. Número de seguidores conectados a Twitter según las horas y días de la semana. Fuente: http://www.tweriod.com/

- Publicación de tweets relacionados con los cursos. Teniendo en cuenta los momentos de máxima visibilidad comentados anteriormente, se ha utilizado Tweetdeck (disponible en: http://tweetdeck.twitter.com), una aplicación de escritorio que permite gestionar varias cuentas a la vez y programar distintas acciones, como tweets y respuestas. De esta manera, se han podido programar tweets que se publicaban automáticamente cuando el número de seguidores conectados era mayor. A través de los hashtag adecuados, se ha dado mayor visibilidad a los cursos y se han enriquecido sus contenidos aportando otras informaciones relacionadas con los mismos, favoreciéndose la interacción con los usuarios. La figura 5 recoge algunos tweets seleccionados de la aplicación Analytics de la página oficial de Twitter, en los que se muestra información de tres tipos: en la primera columna, se ofrecen datos sobre las impresiones, es decir, sobre el número de veces que ha sido visto cada tweet; en la segunda columna, se indica el número de interacciones con otros usuarios mediante retweets, clics en el perfil, etc.; y, en la última columna, se presenta la tasa de interacción en porcentaje.

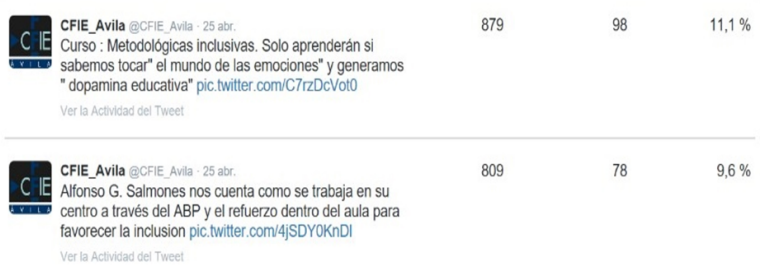

Figura 5. Tweets con indicación de las impresiones y las interacciones. Fuente:

https://analytics.twitter.com/user/CFIE_Avila/tweets

- Utilización de Storify para para publicar resúmenes de los cursos y su difusión a través de Twitter. Storify es una herramienta online de recopilación que permite organizar, en una línea de tiempo, contenidos incorporados desde una gran variedad de redes sociales, como Twitter, Facebook, Instagram, audios de SoundCloud y vídeos. Se ha utilizado para recopilar todas las publicaciones relacionadas con distintos cursos, tal y como puede observarse en el enlace https://goo.gl/XuGZOq, relativo al curso Laboratorio Low Cost, motivando a pequeños grandes científicos, que se muestra como ejemplo.

- App propia del CFIE de Ávila para sistemas Android descargable a través de Twitter. Se ha diseñado una app propia para facilitar el acceso a la información fundamental de los cursos formativos ofrecidos por nuestra institución, como sus plazos de inscripción, los destinatarios prioritarios, el lugar y las fechas de realización (figura 6).

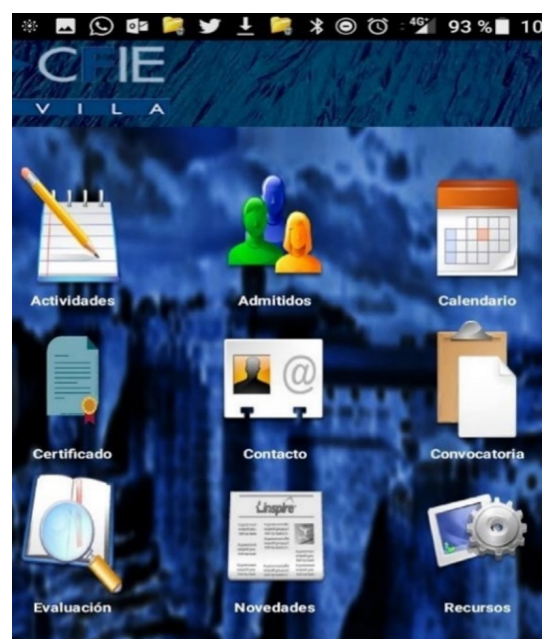

Figura 6. App del CFIE de Ávila para sistemas Android Fuente: Elaboración propia

2.3. Técnicas e instrumentos para la recogida de datos Los resultados positivos de la experiencia se fueron 
Berrón Ruiz, Elena y Régil López, María Victoria (2018). "Twitter como instrumento para fomentar la participación del profesorado en los cursos formativos". @tic revista d'innovació educativa, 20, 43-51.

percibiendo a lo largo de todo el proceso a través de los comentarios que el profesorado nos iba realizando a todo el equipo asesor del CFIE en conversaciones informales. No obstante, se consideró que dichos comentarios no eran suficientemente rigurosos para extraer unas conclusiones significativas. Por ello, se analizaron otros datos que permitieran aportar mayor validez a las conclusiones del estudio, mediante su triangulación. Al respecto, se optó por utilizar tres tipos de técnicas o instrumentos:

- Herramientas analíticas (Analytics, Tweriod y Tweetstats), que han permitido comprobar el alcance de la difusión de los cursos.

- Análisis comparativo de las memorias finales del CFIE de Ávila de los cursos académicos $2015 / 2016$ y $2016 / 2017$, en las que se recogen datos relativos a la participación del profesorado en los cursos presenciales.

- Utilización de un cuestionario cumplimentado por los docentes participantes al finalizar los cursos formativos, en el cual se incluyó una pregunta (la que interesaba en nuestro estudio) que permitía valorar directamente la percepción del profesorado sobre las novedades introducidas para su impulso a través de Twitter. Dicha pregunta era "¿Qué opinas de la información sobre el curso proporcionada a través de Twitter? (marca todas la opciones que consideres conveniente). a). No la he visto; b). Es innecesaria, porque con la información recibida por los cauces tradicionales (díptico enviado a los centros y página web) es suficiente; $c$ ). Me ha resultado útil antes y durante todo su desarrollo; d). Me ha motivado para hacer el curso".

\section{Análisis y discusión de los resultados}

Para facilitar su comprensión, los resultados se presentarán organizados en función de su relación con los dos objetivos planteados en el estudio.

\subsection{Utilidad de las estrategias para dinamizar los cursos en Twitter}

El primer objetivo de la investigación era valorar la utilidad de distintas estrategias para dinamizar los cursos de formación permanente del profesorado en la red social de Twitter. Al respecto, se ha observado que, debido a las mismas, ha aumentado el número de seguidores del CFIE de Ávila en dicha red social. Para comprobarlo, en la figura 7 se recoge la evolución en el número de nuevos seguidores adquiridos cada mes, desde septiembre de 2015 hasta junio de 2017, resultando muy significativo el crecimiento experimentado durante el curso 2016/2017, respecto al anterior 2015/2016. Cabe destacar que, comparando los totales anuales de ambos cursos, se ha obtenido un aumento porcentual del $540 \%$, es decir, dicho valor se ha quintuplicado.

\begin{tabular}{|c|c|c|c|c|c|c|c|c|c|c|c|}
\hline & Sep. & Oct. & Nov. & Dic. & Ene. & Feb. & Mar. & Abr. & May. & Jun. & Total \\
\hline $\begin{array}{l}2015 / \\
2016\end{array}$ & 1 & 3 & 6 & 2 & 4 & 12 & 7 & 3 & 5 & 2 & 45 \\
\hline $\begin{array}{l}2016 / \\
2017\end{array}$ & 39 & 45 & 50 & 16 & 38 & 40 & 32 & 16 & 2 & 10 & 288 \\
\hline
\end{tabular}

Figura 7. Nuevos seguidores de la cuenta de Twitter adquiridos cada mes. Fuente: Elaboración propia
Este resultado va acompañado de un mayor número de visitas al perfil, que es muy superior durante el segundo curso académico, tal y como puede apreciarse en la figura 8. En este caso, comparando los totales anuales de ambos cursos, se ha obtenido un aumento porcentual del $710,26 \%$, es decir, el valor se ha septuplicado.

\begin{tabular}{ccccccccccc|c}
\hline & Sep. & Oct. & Nov. & Dic. & Ene. & Feb. & Mar. & Abr. & May. & Jun. & Total \\
\hline $\mathbf{2 0 1 5 1}$ & 9 & 43 & 258 & 137 & 265 & 278 & 189 & 406 & 307 & 97 & 1989 \\
$\mathbf{2 0 1 6}$ & & & & & & & & & & & \\
$\mathbf{2 0 1 6 1}$ & 444 & 2797 & 3647 & 1069 & 1635 & 1855 & 1525 & 1422 & 1063 & 659 & 16116 \\
\hline $\mathbf{2 0 1 7}$ & & & & & & & & & & & \\
\hline
\end{tabular}

Figura 8. Visitas realizadas al perfil del CFIE de Ávila en Twitter. Fuente: Elaboración propia

En dicha tabla se observa que, durante el curso $2016 / 2017$, el mayor número de visitas se ha producido en octubre, noviembre, enero, febrero y marzo, coincidiendo con los meses en los que se han celebrado más cursos de formación presencial en el CFIE (26 cursos de los 32 que han tenido lugar durante todo el año) $y$, por tanto, coincidiendo también con los momentos en los que se han aplicado más intensamente las nuevas estrategias de difusión referidas en el apartado 2.2 de este artículo.

Asimismo, al comparar los cursos académicos $2015 / 2016$ y 2016/2017, se observan resultados llamativos respecto al número de impresiones de los tweets (figura 9) y de interacciones (figura 10), siendo nuevamente muy superiores durante el segundo año. Al respecto, cabe destacar que el aumento porcentual en el número de impresiones ha sido del 514\%, es decir, se han sextuplicado, y el aumento porcentual en el número de interacciones ha sido del 921,79\%, lo que significa que se han decuplicado.

\begin{tabular}{|lcccccccccc|c}
\hline & Sep. & Oct. & Nov. & Dic. & Ene. & Feb. & Mar. & Abr. & May. & Jun. & Total \\
\hline $\mathbf{2 0 1 5 /}$ & 872 & 1463 & 3784 & 1660 & 2708 & 2981 & 1660 & 5701 & 7004 & 1815 & 29648 \\
$\mathbf{2 0 1 6}$ & & & & & & & & & & \\
\hline $\mathbf{2 0 1 6 /}$ & 2693 & 22100 & 29800 & 15000 & 15500 & 31100 & 16000 & 15600 & 8748 & 5510 & 182051 \\
\hline $\mathbf{2 0 1 7}$ & & & & & & & & & & & \\
\hline
\end{tabular}

Figura 9. Impresiones realizadas en los tweets de los cursos del CFIE de Ávila. Fuente: Elaboración propia

\begin{tabular}{lcccccccccc|c}
\hline & Sep. & Oct. & Nov. & Dic. & Ene. & Feb. & Mar. & Abr. & May. & Jun. & Total \\
\hline $\mathbf{2 0 1 5 /}$ & 8 & 57 & 305 & 10 & 41 & 143 & 66 & 72 & 243 & 74 & 1019 \\
$\mathbf{2 0 1 6}$ & & & & & & & & & & \\
$\mathbf{2 0 1 6 /}$ & 138 & 1525 & 2968 & 530 & 989 & 1751 & 757 & 947 & 669 & 138 & 10412 \\
\hline $\mathbf{2 0 1 7}$ & & & & & & & & & & & \\
\hline
\end{tabular}

Figura 10. Interacciones realizadas en los tweets de los cursos del CFIE de Ávila. Fuente: Elaboración propia

Todos estos datos coinciden con las respuestas ofrecidas por los 527 docentes que cumplimentaron el cuestionario mencionado en el apartado 2.3 (figura 11), ya que, en la pregunta "¿Qué opinas de la información sobre el curso proporcionada a través de Twitter?", el $51,4 \%$ señalaron que dicha información les había resultado útil antes y durante todo su desarrollo (respuesta $\mathrm{C}$ ), lo cual indica que estuvieron pendientes de la misma y que les había interesado. Aunque el porcentaje pudiera parecer poco elevado, se estima que es valioso frente al escaso $8,1 \%$ que consideraron que la información recibida por los cauces tradicionales (carteles, e-mails y página web) ya era suficiente (respuesta B). Igualmente, cabe destacar que el 22,8\% señalaron que no la habían visto (respuesta A), por lo que, proporcionalmente, si comparamos únicamente los resultados de las respuestas $\mathrm{B}$ y $\mathrm{C}$, que son las que han 
Berrón Ruiz, Elena y Régil López, María Victoria (2018). "Twitter como instrumento para fomentar la participación del profesorado en los cursos formativos". @tic revista d'innovació educativa, 20, 43-51.

sido elegidas por los docentes que sí han accedido a la información proporcionada en Twitter, observamos que, de las 314 respuestas ofrecidas en total $(43+271)$, el $86 \%$ la han considerado útil frente al $14 \%$ restante. Por todo ello, se considera que estos porcentajes apoyan la pertinencia de las nuevas estrategias de difusión planteadas en este estudio, además de hacernos reflexionar sobre la importancia de seguir insistiendo para familiarizar al profesorado en la utilización de la red social.

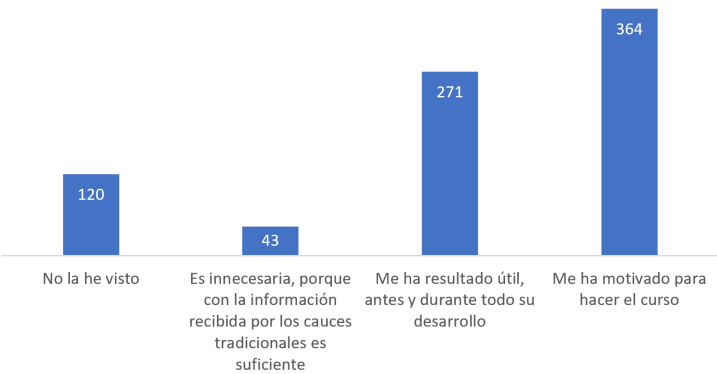

Figura 11. Respuestas ofrecidas a la pregunta del cuestionario "¿Qué opinas de la información sobre el curso proporcionada a través de Twitter?" Fuente: Elaboración propia

\subsection{Análisis del impacto de las estrategias de difusión en la participación}

El segundo objetivo del trabajo era analizar el impacto de la difusión realizada a través de Twitter en la participación del profesorado en los cursos formativos. En relación al mismo, la figura 12 muestra el número de cursos ofertados por el CFIE de Ávila durante los dos últimos años, en función de la demanda, así como los que se realizaron completamente y los que tuvieron que ser suspendidos por no contar con el número mínimo de inscripciones.

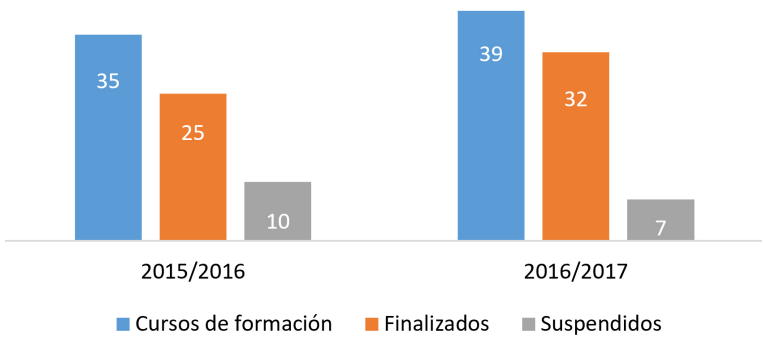

Figura 12. Cursos ofertados por el CFIE, indicando los finalizados y los suspendidos. Fuente: Elaboración propia

De estos datos se desprende que el primer año finalizaron el $71,43 \%$ de los cursos, frente al $82,05 \%$ del segundo, lo cual supone un aumento del 10,62\%. Este aumento podría haber sido incluso mayor porque, durante el año 2016/2017, 5 de los cursos suspendidos tenían 9 participantes inscritos, siendo necesarios 10 para que hubieran podido realizarse.

Por otra parte, la figura 13 recoge el número total de participantes en los cursos e, igualmente, se observa un notable crecimiento durante el año 2016/2017, respecto al anterior 2015/2016, siendo porcentualmente de un $15,21 \%$ más.

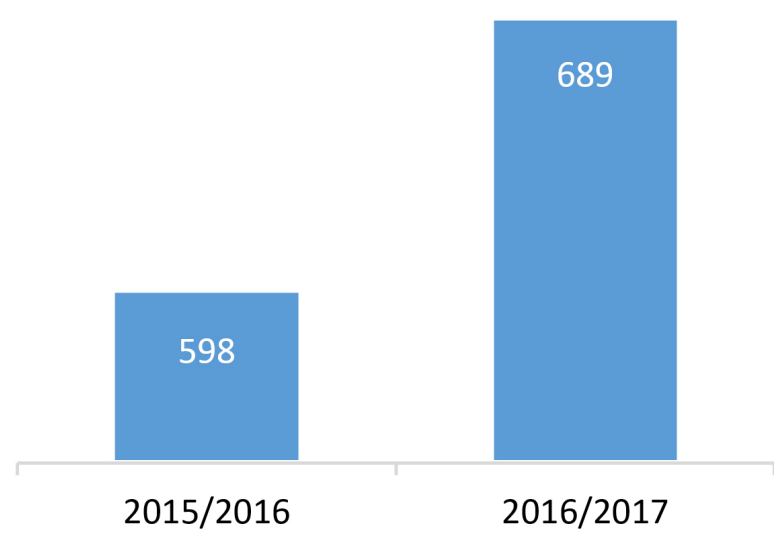

Figura 13. Número de participantes en los cursos organizados por el CFIE de Ávila. Fuente: Elaboración propia

Cabe destacar que el número de cursos ofertados en el curso 2016/2017 es ligeramente superior que en el anterior, lo cual ha podido contribuir al aumento de los participantes. No obstante, si comparamos el aumento porcentual en el número total de participantes (que, como se ha comentado, ha sido del 15,21\%) con el aumento porcentual en las variables analizadas en Twitter (figura 14), observamos resultados muy llamativos y relevantes, ya que el crecimiento en todas las variables de la red social es muy superior, lo cual demuestra que las estrategias desarrolladas han tenido un efecto muy positivo en su fomento.

\begin{tabular}{lc}
\hline \multicolumn{1}{c}{ VARIABLES } & AUMENTO PORCENTUAL \\
\hline $\begin{array}{l}\text { Nuevos seguidores de la cuenta de Twitter } \\
\text { adquiridos cada mes }\end{array}$ & $540 \%$ \\
$\begin{array}{l}\text { Visitas realizadas al perfil del CFIE de } \\
\text { Ávila en Twitter }\end{array}$ & $710,26 \%$ \\
$\begin{array}{l}\text { Impresiones realizadas en los tweets del } \\
\text { CFIE de Ávila }\end{array}$ & $514 \%$ \\
$\begin{array}{l}\text { Interacciones realizadas en los tweets del } \\
\text { CFIE de Ávila }\end{array}$ & $921,79 \%$ \\
\hline
\end{tabular}

Figura 14. Aumento porcentual del curso 2016-2017, respecto al anterior 2015-2016. Fuente: Elaboración propia

Otro dato destacable es que, como muestra la figura 15, durante el año 2016/2017 ha crecido el número de cursos con más de 20 participantes, produciéndose un aumento porcentual del $64 \%$ respecto al anterior año $2015 / 2016$. Estos datos refuerzan igualmente la idea de que las nuevas estrategias de difusión de los cursos han podido favorecer el interés del profesorado para realizarlos.

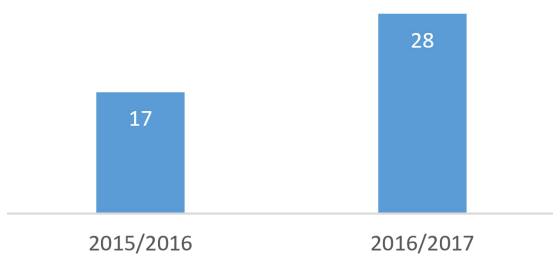

Figura 15. Cursos organizados por el CFIE con más de 20 participantes. Fuente: Elaboración propia

Como las temáticas de los cursos han sido un poco diferentes en cada uno de los años, se podría llegar a pensar que el aumento de participación en los mismos 
Berrón Ruiz, Elena y Régil López, María Victoria (2018). "Twitter como instrumento para fomentar la participación del profesorado en los cursos formativos". @tic revista d'innovació educativa, 20, 43-51.

se debe al mayor interés despertado por los últimos respecto a los que se propusieron el primer año. No obstante, realizando un análisis de las temáticas de los cursos que hubo en el año 2016/2017, encontramos datos muy relevantes. Por un lado, hubo que cancelar 5 cursos que no tuvieron un número suficiente de inscripciones (figura 16) y que, a priori, se esperaba que tuvieran mucha demanda porque sus temáticas coincidían directamente con las líneas prioritarias de formación del profesorado establecidas por la Consejería de Educación de la Junta de Castilla y León y recogidas, igualmente, en la convocatoria provincial de actividades de formación permanente del profesorado de Ávila para el curso 2016/2017.

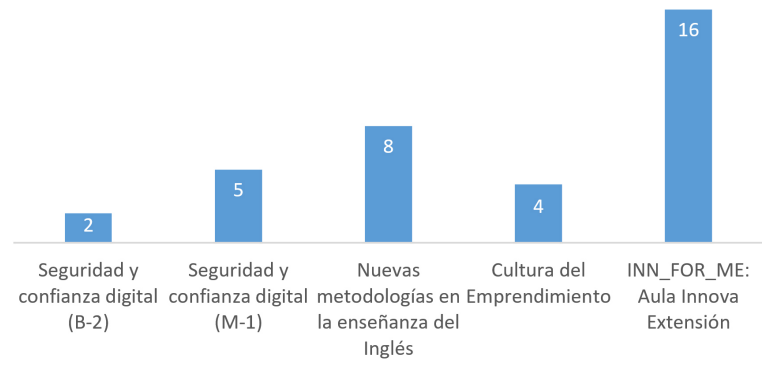

Figura 16. Número de inscritos en cursos prioritarios y promocionados por los canales tradicionales. Fuente: Elaboración propia

Por otro lado, las temáticas de los cursos recogidos en la figura 17 estaban dirigidas a sectores docentes minoritarios y, por ello, se consideró oportuno aumentar su difusión a través de Twitter con las estrategias comentadas en el apartado 2.2. de este artículo, con la finalidad de favorecer la motivación del profesorado. Con ello, se consiguió que el número de inscripciones duplicara el mínimo necesario para poder desarrollarlos. Estos datos refuerzan la idea de que el nuevo impulso dado a los cursos en Twitter ha contribuido decisivamente a la mayor participación de los docentes.

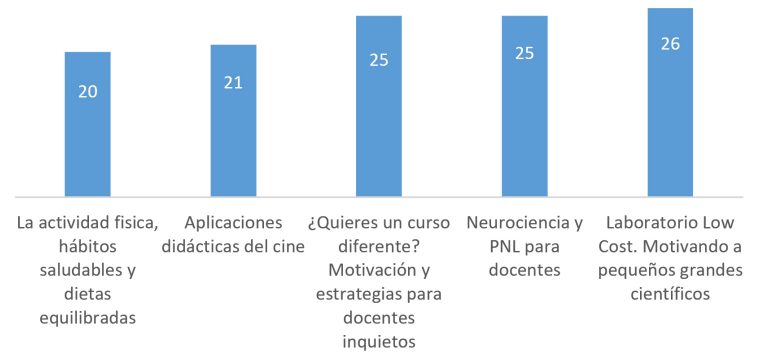

Figura 17. Número de inscritos en cursos dirigidos a sectores minoritarios y promocionados en Twitter. Fuente: Elaboración propia

Finalmente, destacaremos que todos estos datos son coherentes con las respuestas ofrecidas por los profesores en el cuestionario (figura 11), ya que el 69,9\% indicaron que la información proporcionada a través de Twitter les había motivado para hacer los cursos (respuesta D).

\section{Conclusiones y consideraciones finales}

La sociedad está en continua transformación y, actualmente, las redes sociales han cambiado nuestra manera de comunicarnos y de percibir el mundo (Polinario, 2016). La forma de aprender de los alumnos, sus necesidades y circunstancias no son las mismas que hace veinte años, por lo que la organización de la respuesta educativa también debe cambiar (Gómez, 2016). Del mismo modo, las circunstancias particulares de cada centro educativo, así como las características propias de su alumnado y su contexto, justifican la necesidad de formación continua de los docentes a lo largo de toda su trayectoria profesional, pero la oferta de esta formación también debe adecuarse a la realidad del momento (Romero y Trigo, 2015).

En el trabajo presentado en este artículo, que puede definirse como una innovación en proceso, se apostaba por el potencial de las redes sociales como fuente de información y motivación, coincidiendo con las propuestas de Marcos-García (2017). En concreto, como primer objetivo, en el CFIE de Ávila nos interesamos por mejorar la difusión de los distintos cursos ofrecidos al profesorado como parte de su formación permanente, utilizando para ello la red social de Twitter, por las posibilidades comunicativas e interactivas que presenta (Campos-Domínguez, 2017). Al respecto, podemos concluir que los nuevos recursos de difusión de los cursos formativos a través de Twitter utilizados durante el año académico 2016/2017 con respecto al anterior 2015/2016 han despertado gran interés entre el profesorado, favoreciendo su proyección externa, la adquisición de 288 nuevos seguidores de la cuenta del CFIE y el crecimiento de las interacciones e impresiones sobre los mismos, produciéndose un notorio aumento porcentual, del $922 \%$ y del $514 \%$ respectivamente.

Es importante señalar que, en un solo año, se ha pasado de tener 735 seguidores a alcanzar 1023, pero lo que, desde nuestro punto de vista, resulta más destacable es que, a pesar de que en el sector docente se siguen utilizando mayoritariamente los carteles, el correo electrónico y la página web del CFIE para acceder a la oferta formativa, se ha conseguido iniciar al profesorado en el uso de vías de comunicación alternativas a las tradicionales. Con ello, se logra una mayor adaptación a los nuevos tiempos, en los que priman los recursos multimedia y el uso de redes sociales para el acceso a la información (Mas-Bleda y Aguillo, 2015).

El segundo objetivo que se perseguía en este estudio era comprobar si las nuevas estrategias de difusión empleadas a través de Twitter favorecían una mayor participación del profesorado en los cursos, lo cual también se ha conseguido satisfactoriamente, ya que ha habido un notable ascenso respecto al año anterior, en el cual no se practicaban dichas estrategias. Se ha constatado una amplia diferencia en el número de tweets de los cursos suspendidos respecto a los que sí se han celebrado, lo cual parece indicar que la falta de interacción de los cursos en la citada red social es una posible causa del escaso éxito de los mismos (Carpenter y Krutka, 2014). Esta circunstancia se ha dado incluso en cursos que, en principio, se esperaba que tuvieran una gran demanda, ya que su temática coincidía con las líneas prioritarias detectadas por la Consejería de Educación de la Junta de Castilla y León, lo cual evidencia aún más la utilidad de las propuestas de difusión social empleadas.

Por otra parte, es muy significativo el aumento porcentual del 64\% experimentado en el número de cursos con más de 20 participantes. Asimismo, la continua interacción en Twitter durante los cursos formativos ha puesto de manifiesto que la utilización de 
Berrón Ruiz, Elena y Régil López, María Victoria (2018). "Twitter como instrumento para fomentar la participación del profesorado en los cursos formativos". @tic revista d'innovació educativa, 20, 43-51.

hashtag atrayentes aumentaba la participación entre los docentes (Caleffi, 2015). Estos resultados son acordes con las respuestas ofrecidas por los docentes en el cuestionario, ya que la mayoría manifestaron que la información que se ofrecía en Twitter les había resultado útil antes y durante todo su desarrollo e, incluso, el $69,9 \%$ afirmaron que les había motivado para realizar los cursos.

El aumento constatado en la participación e implicación docente en los cursos propuestos nos anima a continuar trabajando en la línea iniciada para mejorar la difusión de los demás procesos formativos. Igualmente, como futura línea de investigación que dé continuidad al trabajo realizado, quizá fuera relevante analizar de qué modo utiliza el profesorado de Ávila la red social de Twitter en el aula, ya que ello permitiría valorar la necesidad de organizar desde el CFIE algún curso específico sobre cómo llevar a cabo un uso educativo eficaz.

Un fin prioritario de nuestro sistema educativo es que los alumnos adquieran una competencia digital acorde a los tiempos actuales (Zabala y Arnau, 2014), y conseguirlo parte de una formación adecuada del profesorado. Aunque en Castilla y León aún no están contempladas las figuras responsables de la gestión de las redes sociales ni sus funciones, hemos observado que algunos docentes están explorando sus potencialidades ejerciendo de community manager de manera autodidacta, mientras que otros se resisten al cambio (Salzano, 2015). Por tanto, como órgano responsable de la formación, consideramos necesario que el CFIE de Ávila continúe abriendo horizontes en los nuevos canales de comunicación para familiarizar al profesorado dependiente de su ámbito de gestión.

\section{Bibliografía}

Abella, V. y Delgado, V. (2015). Aprender a usar Twitter y usar Twitter para aprender. Profesorado: Revista de curriculum y formación del profesorado, 19(1), 364378. Recuperado de: https://goo.gl/i4p59t

Barba, J. J., González-Calvo, G. y Barba-Martín, R. A. (2014). Que la fuerza esté contigo: desvelar el lado oscuro de la investigación en educación. Magis. Revista Internacional de Investigación en Educación, 7(14), 125-140. DOI: https://doi.org/10.11144/javeriana.m7-14.qlfe

Blanco, M., y R. Sueiro (2014): Cómo conseguir 10.000 seguidores en Twitter. Madrid: ESIC.

Bolívar, A., Domingo, J., Escudero, J. M., González, M.T. y García, R. J. (2007). Asesoría Pedagógica. Módulo I: El centro como contexto de formación. Madrid: CENICE.

Caleffi, P. M. (2015). The 'Hashtag': A New Word or a New Rule?. Journal of Theoretical Linguistics, 13(2), 46-69. Recuperado de: http://ir.uv.es/ROOSiMP

Campos-Domínguez, Eva (2017). Twitter y la comunicación política. El profesional de la información, 26(5), 785-793. DOI: https://doi.org/10.3145/epi.2017.sep.01

Carpenter, J. P. y Krutka, D. G. (2014). How and Why educators use twitter: a survey of the field. Journal of Research on Technology in Education, 46(4), 414-
434.

DOI: https://doi.org/10.1080/15391523.2014.925701

Davidson, C. N. y Goldberg, D. T. (2009). The future of learning institutions in a digital age. New York: The MIT Press.

Forkosh-Baruch, A. y Hershkovitz, A. (2012). A case study of Israeli higher-education institutes sharing scholarly information with the community via social networks. The Internet and Higher Education, 15(1), 58-68. DOI: https://doi.org/10.1016/i.iheduc.2011.08.003

Gairín, J. y Armengol, C. (2008). Estrategias de formación para el cambio organizacional. Madrid: Wolters Kluwer Educación.

García Llamas, J. L. (2003). Métodos de investigación en Educación II: Investigación cualitativa y evaluativa. Madrid: UNED.

Gómez, I. M. (2016). La inclusión de las tecnologías en la formación inicial del profesorado: una intervención de aula a través del modelo TPACK. Tendencias Pedagógicas, 28, 133-152. Recuperado de: https://goo.gl/kZELVe

Grosseck, G. (2009). To use or not to use web 2.0 in higher education?. Procedia Social and Behavioral Sciences, $1, \quad 478-482 . \quad$ DOI: https://doi.org/10.1016/i.sbspro.2009.01.087

Grosseck, G. y Holotescu, C. (2011). Teacher education in 140 characters: microblogging implications for continuous education, training, learning and personal development. Procedia Social and Behavioral Sciences, 11, 160-164. DOI: https://doi.org/10.1016/i.sbspro.2011.01.053

Hernández Sampieri, R. (2006). Metodología de la investigación. México: McGrawHill.

Imbernón, F. (2007). Asesorar o dirigir. El papel del asesor/a colaborativo en una formación permanente centrada en el profesorado y en el contexto. REICE. Revista Electrónica Iberoamericana sobre Calidad, Eficacia y Cambio en Educación, 5(1), 145-152. Recuperado de: http://www.redalyc.org/pdf/551/55100108.pdf

Levis, D. (2011). Redes educativas 2.1. Medios sociales, entornos colaborativos y procesos de enseñanza y aprendizaje. Revista de Universidad y Sociedad del Conocimiento (RUSC), 8(1), 7-24. Recuperado de: DOI: http://dx.doi.org/10.7238/rusc.v8i1.965

Manzanares, A. y Galván-Bovaira, M. J. (2012). La Formación Permanente del Profesorado de Educación Infantil y Primaria a través de los Centros de Profesores. Un modelo de evaluación. Revista de Educación, 359(3), 431-455. Recuperado de: http://ir.uv.es/1g40gCV

Marcos-García, S. (2017). El empleo de Twitter como herramienta estratégica de comunicación. Análisis del caso Podemos. Communico, 3, 1-27. Recuperado de: http://ir.uv.es/IVintg6

Mas-Bleda, A. y Aguillo, I. (2015). La Web social como nuevo medio de comunicación y evaluación científica. Barcelona: Editorial UOC.

Pérez-Martínez, V. M., Rodríguez, M. D. y Tobajas, M. (2017). Movilización y participación en Twitter. 
Berrón Ruiz, Elena y Régil López, María Victoria (2018). "Twitter como instrumento para fomentar la participación del profesorado en los cursos formativos". @tic revista d'innovació educativa, 20, 43-51.

Estudio de caso del hashtag \#SuperTuesday en las primarias presidenciales de EEUU 2016. Revista Latina de Comunicación Social, 72, 679-703. DOI: https://dx.doi.org/10.4185/RLCS-2017-1186

Polinario, J. (2016). Cómo divulgar ciencia a través de las redes sociales. Almería: Círculo Rojo

Ramírez, A. (2015). La formación del profesorado en educación primaria ante las competencias básicas. International Electronic Journal of Teacher Training, 52(18), 199-214.

DOI: http://dx.doi.org/10.6018/reifop.18.3.193811

Rodríguez Izquierdo, R. M. (2011). Repensar la relación entre las TIC y la enseñanza universitaria: problemas y soluciones. Profesorado: Revista de Currículum y Formación del Profesorado, 15(1), pp. 9-22. Recuperado de: http://www.ugr.es/ recfpro/rev151ART1.pdf

Romero, M. y Trigo, E. (2015). Herramientas para el éxito, Cuadernos de Pedagogía, 458, 1-5.

Salzano, D. (2015). Repensar la educación en la era de los medios sociales. Hachetetepé: Revista Científica de Educación y Comunicación, 11, 79-90. Recuperado de: https://goo.gl/HECju6
Stake, R. E. (1998). Investigación con estudio de casos. Madrid: Morata.

Stringer, E. T. (2007). Action Research in Education. Upper Saddle River: Pearson.

Trigueros, F.J., Sánchez, R., y Vera, M.I. (2012). El profesorado de Educación Primaria ante las TIC: realidad y retos. Revista Electrónica Interuniversitaria de Formación del Profesorado, 15(1), 101-112. Recuperado de: https://goo.gl/HyxwoM

Zabala, A. y Arnau, L. (2014). Métodos para la enseñanza de las competencias. Barcelona: Graó.

| Cita recomendada de este artículo

Berrón Ruiz, Elena; Régil López, María Victoria (2018). “Twitter como instrumento para fomentar la participación del profesorado en los cursos formativos”. @tic revista d'innovació educativa, 20, 43-51. 